\title{
Energy landscapes of ion clusters in isotropic quadrupolar and octupolar traps
}

\author{
F. Calvo, ${ }^{1, \text { a) }}$ E. Yurtsever, ${ }^{2}$ and D. J. Wales ${ }^{3}$ \\ ${ }^{1}$ LASIM, Université Claude Bernard Lyon 1 and CNRS UMR 5579, 43 Bd du 11 Novembre 1918, \\ F69622 Villeurbanne Cedex, France \\ ${ }^{2}$ Koç University, Rumelifeneriyolu, Sariyer, Istanbul 34450, Turkey \\ ${ }^{3}$ University Chemical Laboratories, Lensfield Road, Cambridge CB2 1EW, United Kingdom
}

(Received 4 November 2011; accepted 8 December 2011; published online 9 January 2012)

\begin{abstract}
The energy landscapes of ion clouds confined in isotropic quadrupolar and octupolar traps are characterized for several representative cluster sizes. All clusters exhibit stable multishell structures that belong to separate funnels. Quadrupolar confinement leads to more homogeneous clusters and denser distributions of isomers than octupolar confinement. Statistical analysis of the transition states indicates that the barriers associated with intrashell motion are lower but more asymmetric and more cooperative compared to intershell motion. The relaxation between low-energy funnels with different arrangements of shells mostly exhibits Arrhenius kinetics, with a weak variation of the activation energy at higher temperatures. (C) 2012 American Institute of Physics. [doi:10.1063/1.3673318]
\end{abstract}

\section{INTRODUCTION}

Atomic ions can be confined into clusters by the application of suitable nonuniform external electromagnetic fields. Devices such as Paul or Penning traps have been shown to contain ions over macroscopically long times under appropriate vacuum conditions, offering a unique way to study the properties of matter bound by long-range forces. ${ }^{1,2}$ Using laser techniques, the clusters can even be cooled down to temperatures that induce spontaneous crystallization into various geometric arrangements. ${ }^{3-6}$ Besides promising applications in metrology, ${ }^{7,8}$ the stable assemblies of trapped ultracold ions are one of the few practical realizations ${ }^{9,10}$ of three-dimensional Wigner crystals, in which finite size effects are strongly manifested. ${ }^{11,12}$

So far, most investigations of ion clusters have been carried out in quadrupolar (harmonic) traps. In the isotropic limit, the corresponding crystals exhibit concentric spherical shells $\mathrm{s}^{5,13,14}$ and undergo a progressive transition towards the bulk body-centered cubic Wigner crystal structure above several thousand ions..$^{15}$ Many experimental ${ }^{5,13,14,16}$ and theoretical ${ }^{11,12,15,17-20}$ studies have been devoted to the structure and thermodynamics of such systems, and it was generally shown that intershell rotations precede melting, ${ }^{11,21}$ a phenomenon also encountered in twodimensional clusters. ${ }^{22,23}$ The different roles played by intrashell and intershell motions were interpreted by Wales and Lee $^{24}$ who identified different categories of transition states for representative clusters.

Higher-order traps have a practical advantage over harmonic confinement: the field-free regions near the center allow more ions to be stored, with noteworthy applications in studies of chemical reactivity. ${ }^{25}$ The possibility of storing and

a) Author to whom correspondence should be addressed. Electronic mail: fcalvo@lasim.univ-lyon1.fr. crystallizing ion clusters in higher order traps has been explored theoretically ${ }^{26-29}$ and, only recently, experimentally. ${ }^{30}$ Among the differences from harmonic traps, the hollow nature of the resulting clusters is probably the most remarkable. Although they still form shells at low temperature, ion crystals in multipolar traps exhibit increasing density with increasing radial distance, ${ }^{26}$ giving rise to unusual core premelting effects upon heating. ${ }^{28}$ Another difference is in the shell capacity, the maximum number of particles a shell can accommodate before the formation of a new shell is favored. Because the lower field at the center does not hold the ions as tightly, the shell capacities grow with the order of the trap, hence for a given number of ions more shells are formed for the quadrupolar case. ${ }^{28,29}$

The present work aims to further examine the structural properties of ion clouds in different types of confinement, by following the lines of previous work by Wales and Lee, but extending this study into a more complete perspective of the energy landscape. This approach ${ }^{31}$ is convenient for understanding the statistical and kinetic properties, using a discrete description based on the important stationary points, namely the local minima and first-order saddles. ${ }^{32,33}$ As will be shown below, the energy landscape reveals distinct shell organization in the quadrupolar and octupolar confinements, suggesting different relaxation mechanisms. However, generic features can be identified for the intra- and intershell transitions in terms of barrier heights, asymmetries, and cooperativities. Our results indicate that ion clouds in higher order traps have more structured landscapes with stronger energetic separation between different shell arrangements, making them poorer structure seekers than their analogues in quadrupolar traps. Comparison with Morse clusters shows that these results are a manifestation of the long range of both the Coulomb interaction and the confinement potential.

Section II briefly describes the models and the computational tools used to sample the energy landscape. 
Section III presents and discusses the results, comparing three cluster sizes for quadrupolar and octupolar isotropic confinement. Section IV provides some discussion and conclusions.

\section{MODELS AND METHODS}

Although isotropic quadrupolar traps can be experimentally realized, ${ }^{14}$ higher order confinement is practically achieved by a number of parallel axial electrodes, confinement along the axis remaining quadrupolar. Experimental octupolar traps are thus not fully isotropic, and ion clusters in such fields adopt hollow cylindrical shapes. ${ }^{27,30,34}$ However, the present work is aimed at identifying general trends as the order of confinement is varied, especially the role of confinement on the energy landscape and shell arrangements. We therefore consider a set of $N$ identical ions confined by an isotropic radial potential $V_{\text {trap }}(r)=A r^{p}$, where $r$ is the distance between the ion and the center of the trap, $A$ is a strictly positive parameter and the exponent $p$ equals 2 or 6 for quadrupolar and octupolar confinements, respectively. We will refer to the corresponding clusters of $N$ ions as $\mathrm{Q}_{N}$ and $\mathrm{O}_{N}$. Neglecting the micromotion arising in radiofrequency traps, the total potential energy felt by the ions at configuration $\mathbf{R}=\left\{\mathbf{r}_{i}\right\}$ reads

$$
V(\mathbf{R})=\sum_{i} A r_{i}^{p}+\sum_{i<j} \frac{q^{2}}{4 \pi \varepsilon_{0} r_{i j}},
$$

where $r_{i j}$ is the distance between ions $i$ and $j, q$ is the charge of the ions, and $\varepsilon_{0}$ is the dielectric permittivity of vacuum. A straightforward change of units leads to the dimensionless equation,

$$
V(\mathbf{R})=\sum_{i} r_{i}^{p}+\sum_{i<j} \frac{1}{r_{i j}} .
$$

We take the center of the trap to be fixed at the origin of the reference frame. The fixed origin breaks translational invariance, so that the dynamical matrix has only three vanishing eigenvalues at each stationary point, corresponding to overall rotations about the origin.

For a few selected cluster sizes $N$, we first searched for the most stable structures adopted in quadrupolar and octupolar traps, using the basin-hopping global optimization method. ${ }^{35,36}$ Databases of connected minima were then constructed using successive doubly nudged ${ }^{37}$ elastic band ${ }^{38-40}$ transition state searches for unconnected minima chosen according to the missing-connection algorithm. ${ }^{41}$ Images corresponding to local maxima were tightly converged to transition states using hybrid eigenvector-following, ${ }^{38,42,43}$ with a root mean square gradient tolerance of $10^{-7}$ for the gradient in reduced units. Expanding stationary point databases in this way forms the basis of the discrete path sampling (DPS) procedure ${ }^{44-46}$ which is designed to provide a representation of the overall kinetics. The OPTIM program ${ }^{47}$ was used for all geometry optimizations and the parallel searches were organized with the PATHSAMPLE program. ${ }^{48}$ More details of the DPS approach can be found in previous accounts. ${ }^{50}$

Transition state theory was used to estimate the rate constants associated with the forward and backward paths cor- responding to each transition state, at fixed temperature $T$. These individual rates require the knowledge of the classical densities of states, which were calculated in the harmonic approximation from the vibrational frequencies at the stationary points. Anharmonic corrections, which could be incorporated through perturbation theory, ${ }^{49}$ are not expected to change the results qualitatively. Rate constants associated with pathways between distant minima, or between sets of minima defined according to a suitable lumping procedure,$^{50}$ were obtained using the DPS method. ${ }^{44}$

\section{RESULTS AND DISCUSSION}

Three cluster sizes were chosen for the two types of confinement, namely $N=90,108$, and 164 . Below 90 ions, octupolar clusters exhibit only a few local minima; the larger sizes considered here are more interesting. The number of distinct local minima for $\mathrm{Q}_{164}$ is reasonably large, and the landscape is more difficult to sample efficiently. Between these limits, the clusters exhibit increasing complexity, as manifested by different numbers of shells. To simplify the descriptions, we refer below to ion clouds under different confinements as quadrupolar or octupolar clusters.

The complexity of a many-particle system is partly reflected in the number of distinct local minima that it supports, and for the present ionic clusters lower order traps permit many more such arrangements. The order of the trap here plays a role similar to the range of the potential, which drastically influences the number of stable configurations. ${ }^{51-53}$ The number of isomers obtained for $\mathrm{Q}_{90}$ and $\mathrm{O}_{90}$ were 926 and 251, for $\mathrm{Q}_{108}$ and $\mathrm{O}_{108}$ were 8189 and 1850, and for $\mathrm{Q}_{164}$ and $\mathrm{O}_{164}$ were 15930 and 9328 , respectively. These values should of course be taken as lower bounds for the total number of local minima, not including permutation-inversion isomers.

For all clusters, the isomers can be categorized according to their shell arrangement, and we use the simple notation $\left(m_{1}\right.$, $\left.m_{2}, \ldots, m_{k}\right)$ to designate an ordered arrangement of $k$ shells with $m_{1}$ ions in the central shell, $m_{2}$ in the second shell, and $m_{k}$ in the outermost shell. Single-shell arrangements, which occur for the octupolar clusters, are thus denoted $(N)$. The shell arrangements found for the most stable structures are generally comparable to results available in the literature, obtained from global optimization ${ }^{11,19}$ or from semi-analytical models. ${ }^{29}$ However, the lowest-energy conformation of the 108 -ion octupolar cluster was found to be a $(2,106)$ arrangement, instead of the single shell pattern predicted by the shell model. ${ }^{28,29}$ Repeating global optimization in the range $N=100-108$ reveals that the two-shell arrangement actually becomes the most stable already for 105 ions, at variance with the predictions of the shell model. Such a discrepancy between the semi-empirical shell model and the numerically exact global optimization result is not exceptional, even though its statistical occurrence was quantified to be below $0.5 \%$ in quadrupolar clusters containing up to 400 ions. ${ }^{54}$ It would be interesting to determine whether shell models perform as well in higher-order traps.

The individual transition states were sorted into various classes depending on the types of shell arrangements they connect. In particular, it is possible to distinguish transition 
(a)

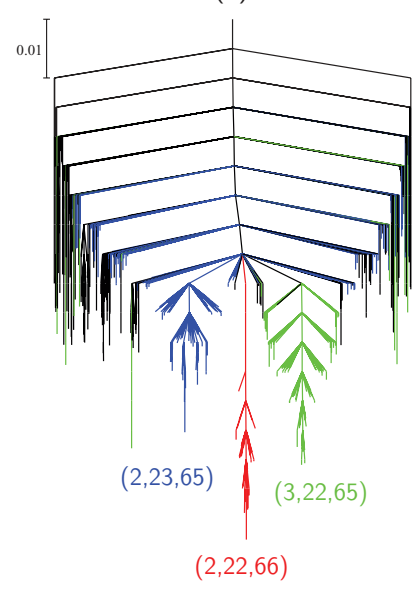

(b)

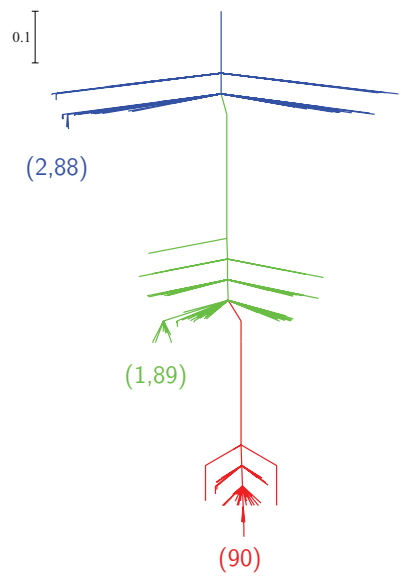

FIG. 1. Disconnectivity graphs obtained for 90-ion clusters in (a) a quadrupolar trap and (b) an octupolar trap. The shell arrangements are indicated by numbers in parentheses and by the coloring scheme. The energy scales are indicated by vertical bars.

states corresponding to intrashell motion, where the minima have the same shell structure, from transition states that connect different shell arrangements.

The stationary points and their connections are best visualized by plotting disconnectivity graphs, ${ }^{55}$ which show how the minima of the landscape are connected to each other and what are the heights of the barrier separating them. In these graphs, each minimum starts from a vertical line at the corresponding energy, and is connected to other minima at the transition state energies, the latter being grouped for clarity. Finite temperature $^{56}$ and zero-point vibrational ${ }^{57}$ effects can also be incorporated by replacing the energies of the stationary points by their classical or quantum free energies, using harmonic or anharmonic ${ }^{58}$ approximations for the individual partition functions. Further details on disconnectivity graphs can be found in Ref. 31.

In the case of trapped ion clusters, we use separate colors to distinguish the most stable shell arrangements. Low-energy parts of the disconnectivity graphs are depicted in Figs. 1-3 for the 90-, 108-, and 164-ion clusters, respectively. $\mathrm{Q}_{90}$ is most stable in the $(2,22,66)$ pattern, but competing arrangements exist with $(3,22,65)$ and $(2,23,65)$ shells.

In the octupolar case, the most stable configuration for 90 ions has a single shell, in agreement with previous work. ${ }^{28,29}$ However, the two-shell arrangements $(1,89)$ and $(2,90)$ are also found, although at significantly higher energies. Removing one ion and placing it off center is not allowed in shell models for higher-order traps, but is a clear (and even competitive) possibility in the present, ion-resolved systems.

For the two types of confinement, each shell arrangement appears as a distinct funnel in the disconnectivity graph, with relatively small barriers within each funnel and larger barriers between funnels. Such landscapes thus display a clear separation of energy scales between intra- and intershell mechanisms, as anticipated in earlier work. ${ }^{24}$

For 90 ions, the two side funnels found for the quadrupolar cluster suggest that relaxation to equilibrium will be faster for the octupolar trap. However, as expected for finite (a)

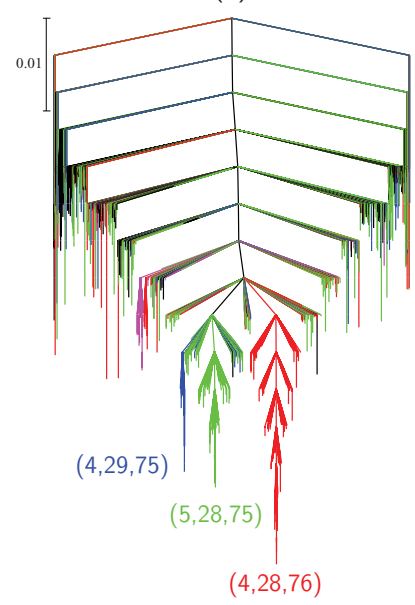

(b)

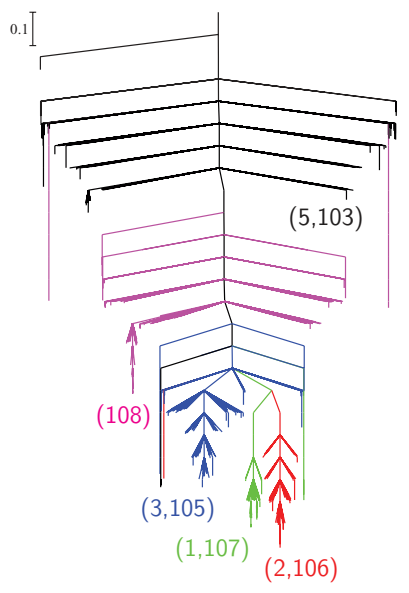

FIG. 2. Disconnectivity graphs obtained for 108-ion clusters in (a) a quadrupolar trap and (b) an octupolar trap. The shell arrangements are indicated by numbers in parentheses and by separate colors. Only the lowest 1500 connected minima are represented, and the energy scales are indicated by vertical segments.

clusters, ${ }^{59}$ the landscapes are strongly size-dependent and the disconnectivity graphs obtained for the 108-ion systems show marked differences from $\mathrm{Q}_{90}$ and $\mathrm{O}_{90}$.

$\mathrm{Q}_{108}$ is still most stable as a three-shell configuration, each arrangement being associated with a distinct funnel in the disconnectivity graph. As mentioned above, the lowestenergy minimum of the octupolar cluster turns out to be a $(2,106)$ configuration. Even though global optimization results differ from the predictions of the shell model, ${ }^{28,29}$ $N=108$ remains close to the structural transition in octupolar traps, with the appearance of a second shell. This proximity is manifested by the larger number of coexisting funnels associated with different shell arrangements, where the number of ions in the inner shell varies from 0 to 5 (Fig. 2). Except for the $(5,103)$ arrangement, the other five funnels are fairly close to each other in energy and correspond to slower relaxation time scales.

Under quadrupolar and octupolar confinement, the 164ion clusters prefer to form four and two shells, respectively. Only two side funnels have clear signatures in the disconnectivity graph of the quadrupolar system, with one or two additional ions in the second shell at the expense of the outermost shell. As for the other landscapes obtained for this confinement, the corresponding graph exhibits some frustration, in the form of low-lying minima separated by relatively high barriers. $^{60}$

The octupolar cluster shows three two-shell arrangements with distinct energies, the global minimum lying at the bottom of its own separate funnel. However, isomers corresponding to a particular shell arrangement may be connected only via high barriers, resulting in the "layered" graph of Fig. 3(b).

The stronger dependency of potential energy on the shell occupancies in the octupolar case thus generally leads to disconnectivity graphs with a more marked hierarchical character. The correspondence between the landscapes and the intra- or intershell nature of the transition states 
(a)

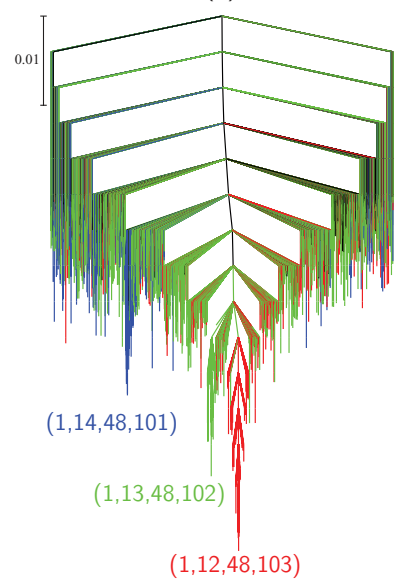

FIG. 3. Disconnectivity graphs obtained for 164-ion clusters in (a) a quadrupolar trap and (b) an octupolar trap. The shell arrangements are indicated by numbers in parentheses and by separate colors. Only the lowest 1500 connected minima are shown, and the energy scales are indicated by vertical segments.

also partly emerges from the disconnectivity graphs. Different shell occupancies essentially correspond to specific funnels, and the barriers within a specific shell arrangement are therefore lower than those separating distinct shell arrangements. This result indicates that, for both confinements, intrashell motion should be much easier than intershell motion.

A more detailed investigation of the properties of the transition states is required to confirm these ideas on a rigorous basis. The databases built for the quadrupolar clusters probably include only a subset of all the possible transition states, amounting to 6091, 45 099, and 34786 members for increasing cluster size. Despite having fewer transition states, the database obtained for the 164-ion quadrupolar cluster yields a disconnectivity graph that is well converged, at least in the low energy range. The corresponding databases for the octupolar clusters contain 1972, 11 149, and 12536 transition states.

As for the local minima, individual transition states can be sorted according to the shell arrangements of the minima they interconnect. Several such properties were calculated from the databases of stationary points. The average barrier height $E^{\dagger}$ is simply obtained as the arithmetic average between barriers $E^{+}$and $E^{-}$corresponding to the forward and reverse reactions, respectively. The parameter $\beta=\left|E^{+}-E^{-}\right| / \max \left(E^{+}, E^{-}\right)$provides a measure of the barrier asymmetry. This index lies in the range $0 \leq \beta \leq 1$. Following Trygubenko and Wales ${ }^{61}$ we also define a cooperativity index $\widetilde{N}$ from the displacement vectors $\mathbf{r}_{i}^{\mathrm{S}}$ and $\mathbf{r}_{i}^{\mathrm{F}}$ of the particles between their starting and finishing geometries as

$$
\widetilde{N}=\frac{\left[\sum_{i}\left|\mathbf{r}_{i}^{\mathrm{S}}-\mathbf{r}_{i}^{\mathrm{F}}\right|^{2}\right]^{2}}{\sum_{i}\left|\mathbf{r}_{i}^{\mathrm{S}}-\mathbf{r}_{i}^{\mathrm{F}}\right|^{2}}
$$

We also quantified the path length asymmetry from the normalized difference in integrated path length, ${ }^{61}$ but this index turned out not to separate the various types of transition states particularly well.
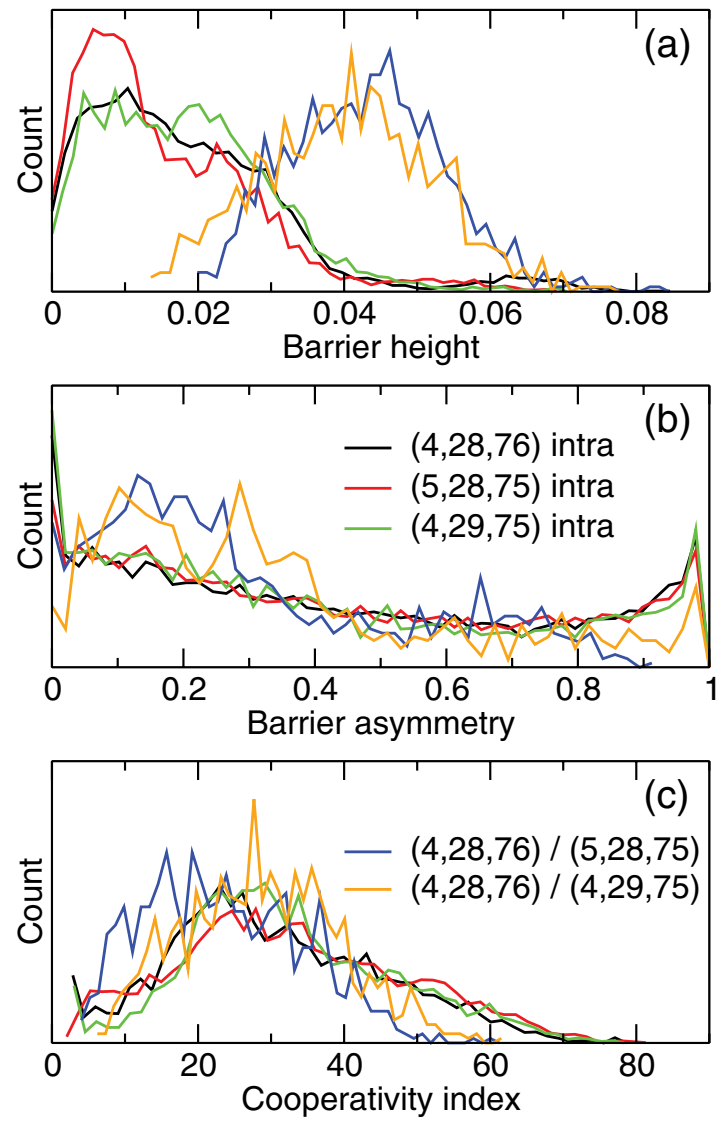

FIG. 4. Distributions of barrier properties associated with the transition states of the 108-ion cluster in a quadrupolar trap, and involving the three lowest-energy funnels of the $(4,28,76),(5,28,75)$, and $(4,29,75)$ shell arrangements. (a) Barrier height; (b) barrier asymmetry; and (c) cooperativity index, all in reduced units.

The 108-ion clusters were chosen to illustrate the generic features associated with intra- and intershell transitions in both types of confinement, focusing on the connections between low-energy funnels. The probability distributions of the parameters $E^{\dagger}, \beta$, and $\widetilde{N}$ are illustrated in Fig. 4 for the quadrupolar cluster and the transitions involving the most stable $(4,28,76)$ arrangement and the $(5,28,75)$ and $(4,29,75)$ secondary funnels.

Strikingly, the barrier height distributions do not depend significantly on the details of the shell arrangements, but mostly on whether the associated motion is inter- or intrashell. These distributions are rather broad for all types of transition state, centered on low values for intrashell rearrangements, but markedly shifted to higher values for barriers in which ions move between shells. Not surprisingly, the barriers corresponding to the simultaneous displacement of two ions from the internal and external shell leaving the central shell at constant occupancy are slightly higher than the barriers connecting the $(4,29,75)$ and $(5,28,75)$ funnels.

The barriers for intrashell motion are mostly evenly distributed in terms of asymmetry, the entire range $0 \leq \beta<1$ being covered, but with two significant peaks at very low and very high $\beta$. This structure indicates a broad variety of transitions. In contrast, the distributions obtained for intershell transitions are shifted toward weaker asymmetries, showing 

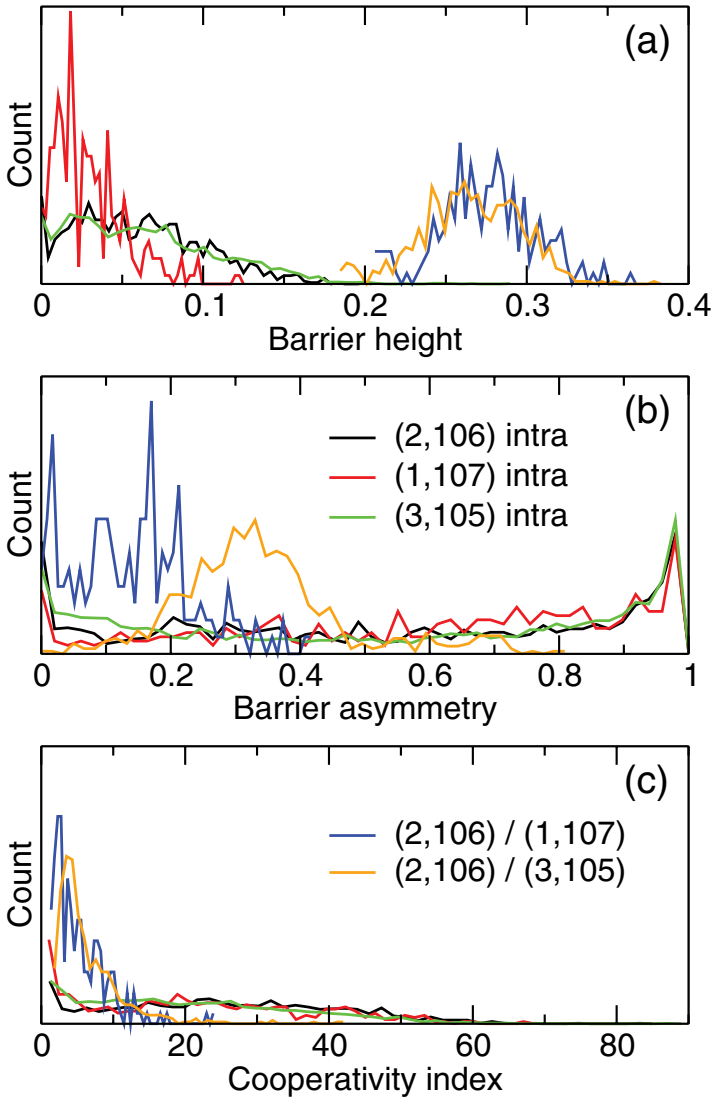

FIG. 5. Distributions of barrier properties associated with the transition states of the 108-ion cluster in octupolar trap, and involving the three lowestenergy funnels corresponding to the $(2,106),(1,107)$, and $(3,105)$ shell arrangements. (a) Barrier height; (b) barrier asymmetry; and (c) cooperativity index, all in reduced units.

that these transitions separate isomers that are closer in energy.

The cooperativity of the transitions is distributed around $\widetilde{N} \approx 20$ for intershell motion, and $\widetilde{N} \approx 25$ for intrashell motion. Ion exchange between shells thus involves fewer particles in the latter case, which can be understood by noting that intrashell transitions have contributions in which the various shells mostly rotate around their common center along different directions, giving a higher $\widetilde{N}$ but also low $E^{\dagger}$ and $\beta$. Cooperativity in these clusters bound by long-range forces is significantly lower than in systems bound by short-range interactions. ${ }^{62,63}$

The statistical differences between transitions associated with intra- and intershell motion are even stronger in the octupolar case, as shown in Fig. 5 for the three most stable arrangements between the $(2,106),(1,107)$, and $(3,105)$ configurations. Because there are fewer transitions within the $(1,107)$ arrangement, the corresponding distributions are slightly more noisy. With respect to the quadrupolar system, the barrier heights for intershell motion are significantly shifted to much higher values, intrashell transitions remaining distributed in the low-energy spectrum (about one order of magnitude difference). The intershell barriers are clearly more symmetric, and much less cooperative than intrashell barriers.

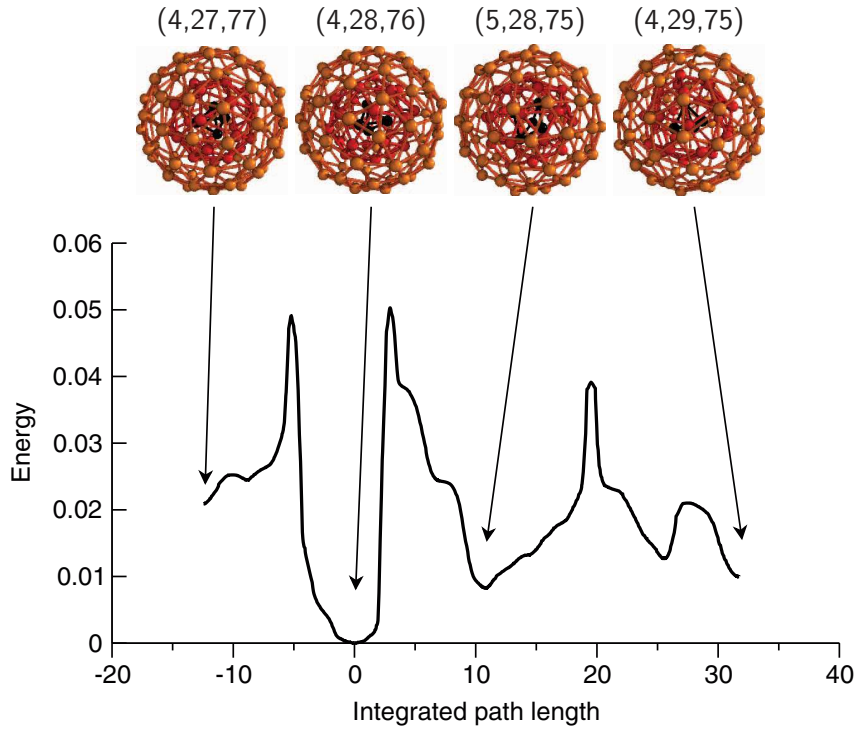

FIG. 6. Pathway connecting the lowest-energy isomers corresponding to various shell arrangements of the 108-ion quadrupolar cluster. All units are reduced.

Hence the general trends observed for quadrupolar confinement are confirmed and even amplified in the octupolar trap. The present results confirm those previously obtained by Wales and Lee, ${ }^{24}$ and are consistent with the observation of intershell rotational motion prior to melting in simulations. ${ }^{11,21}$ In octupolar and higher-order confinements, this effect is magnified and this is also in correspondence with the core premelting effect reported in previous work. ${ }^{28}$

We have also identified low-energy pathways connecting the most stable conformers of the various shell arrangements for the 108-ion clusters. These paths correspond to the largest contribution to the rate constant when intervening minima are placed in steady state. ${ }^{44}$ They were obtained using Dijkstra's algorithm, ${ }^{64}$ as implemented in the PATHSAMPLE program. $^{48}$

For the quadrupolar trap, one such pathway is depicted in Fig. 6 as a function of integrated path length. It connects the $(4,28,76)$ global minimum to the $(4,27,77)$ funnel on one side, and to the $(5,28,75)$ and $(4,29,75)$ funnels on the other side.

The barriers for these rearrangements are rather narrow and high, with values close to about 0.05 energy units, falling in the middle of the distributions of Fig. 4(a). Alternative pathways involving two successive single-ion changes from $(4,28,76)$ to $(4,29,75)$, then into $(5,28,75)$ look very similar.

Likewise, the pathway connecting the six most stable configurations of the octupolar 108-ion cluster, and contributing the most to the rate constant, is depicted in Fig. 7. Again this pathway has two sides leading to the filling of the outer shell in the $(2,106) \rightarrow(1,107) \rightarrow(108)$ fashion, or to the progressive filling of the inner shell according to $(2,106) \rightarrow(3,105) \rightarrow(4,104) \rightarrow(5,103)$. The barriers are close to 0.3 energy units, in agreement with Fig. 5(a). As for the quadrupolar cluster, the saddle points are generally associated with narrow barriers, while the pathway is relatively flat near the minima. This pattern contrasts with results obtained for clusters bound by short-range potentials, ${ }^{63}$ where 


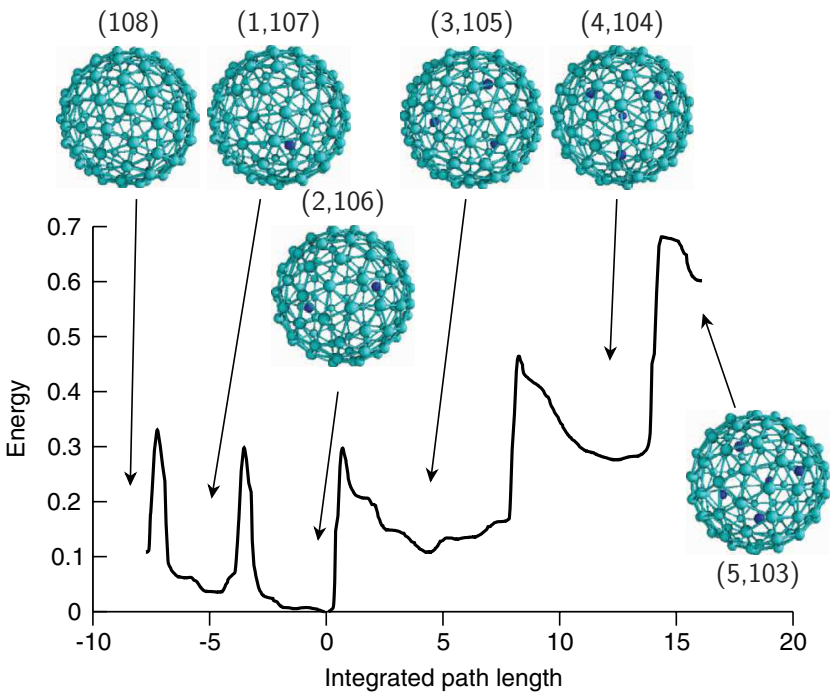

FIG. 7. Pathway connecting the lowest-energy isomers corresponding to various shell arrangements of $\mathrm{O}_{108}$. All units are reduced.

the energy profiles are generally relatively flat near barriers but steep near minima.

The forward and backward rate constants for selected rearrangements have been calculated as a function of the canonical temperature. They are illustrated as Arrhenius plots in Fig. 8 for the two rearrangements $(4,29,75) \rightleftharpoons(4,28,76) \rightleftharpoons(5,28,75)$, grouping all minima that belong to the same shell arrangement or distinguishing the contribution from the pathway involving only the most stable minima. At sufficiently low temperature $T<1 / 500$ and for both rearrangements, the rate constants are dominated by the contribution from the most stable configurations and show regular exponential variations with increasing inverse temperature. However, the activation energy measured by the slope increases slightly at low inverse temperature, a feature also found in moderately fragile glass formers. ${ }^{65}$ Taking into ac-
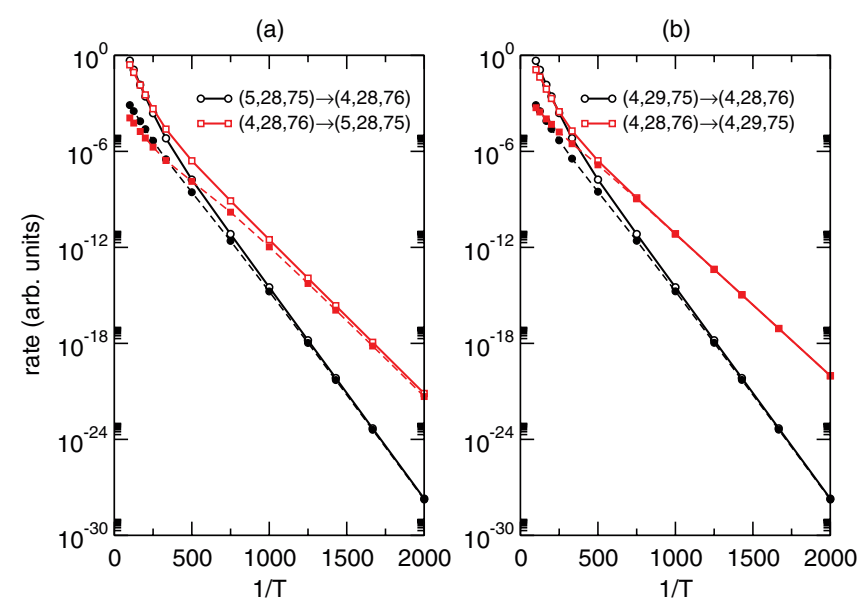

FIG. 8. Rate constants for the transition between low-energy funnels of $\mathrm{Q}_{108}$, shown as Arrhenius plots. (a) Transition between the $(4,28,76)$ and $(5,28,75)$ funnels and (b) transition between the $(4,28,76)$ and $(4,29,75)$ funnels. The rates are shown for the most stable isomers as solid lines with empty symbols, and for the entire interfunnel transitions by summing over the corresponding isomers, as dashed lines with full symbols. All units are reduced. (a)

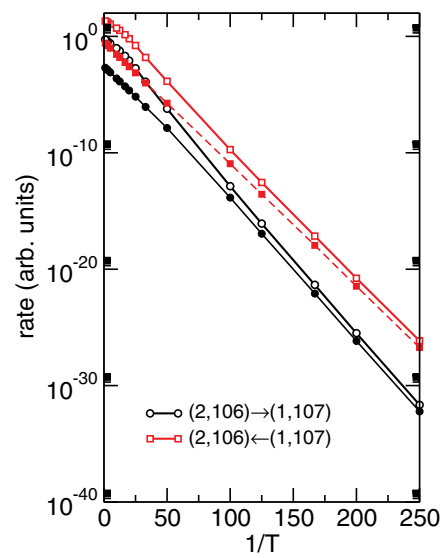

(b)

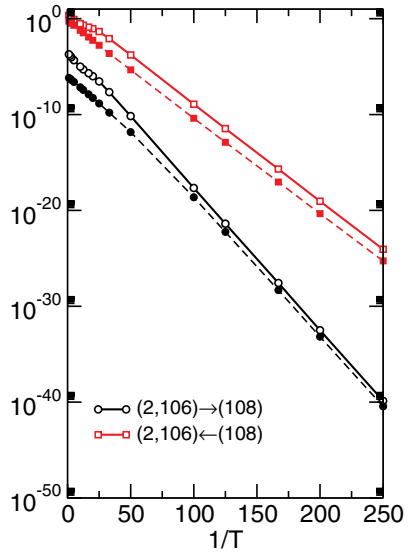

FIG. 9. Rate constants for the transition between low-energy funnels of $\mathrm{O}_{108}$, shown as Arrhenius plots. (a) Transition between the $(2,106)$ and $(1,107)$ funnels and (b) transition between the $(2,106)$ and (108) funnels. The rates are shown for the most stable isomers as solid lines with empty symbols, and for the entire interfunnel transitions by summing over the corresponding isomers, as dashed lines with full symbols. All units are reduced.

count all relevant isomers in the integrated rate constant reduces this effect significantly.

As the temperature exceeds about $1 / 200$, the transitions starting from the most stable $(4,28,76)$ funnel become slower than the backward transitions. This temperature is close to the melting point of such clusters, ${ }^{11,12}$ and the inversion between the forward and backward rates is the likely consequence of a change of stability between the corresponding funnels.

Rate constants have also been calculated for the $(2,106) \rightleftharpoons(1,107)$ and $(2,106) \rightleftharpoons(108)$ rearrangements of the octupolar cluster. The variations of the forward and backward rates are illustrated in Fig. 9 as a function of inverse temperature.

These Arrhenius plots show that the kinetics mostly follow simple first-order relaxation behavior with constant activation energy. The deviations from linearity that appear at high temperatures $T>1 / 50$ lead to lower effective activation energies, at variance with the quadrupolar case. The overall interfunnel rates, although still dominated by the pathways between the most stable conformers, include larger contributions from higher-lying minima and alternative pathways.

Interestingly, the relaxation kinetics in systems bound by short-range forces also display deviations with respect to conventional Arrhenius behavior, ${ }^{63}$ but in the opposite direction with a decreasing activation energy at high temperatures. This behavior, reminiscent of a "fragile" glass former, ${ }^{65}$ is thus also related to the range of the potential.

\section{CONCLUDING REMARKS}

Ion clouds trapped by electromagnetic fields form complex structures, whose detailed geometries are intimately related to the geometry of the trap. In small clusters containing only a few hundred ions, the layering taking place at low temperature appears as a series of thin spherical shells under isotropic confinement. The shell occupancies primarily depend on the order of the trap and on the number of ions, 
and for the most stable structures they can be predicted quite accurately by semi-analytical models. ${ }^{18,28,29}$ However, as with other many-particle systems, numerous isomers are possible, either within a specific type of shell arrangement, or by moving ions to different shells. The dynamics and thermodynamics of these ion assemblies at finite temperature crucially depend on the structure of the local minima and their interconnections.

The purpose of the present work was to examine these issues from the point of view of the energy landscape. The databases of connected minima obtained by successive double-ended searches were visualized using disconnectivity graphs. The different shell arrangements were found to be associated with distinct funnels separated by high barriers, whereas isomerizations that preserve the shell arrangement involve significantly lower barriers. This feature, generic to both quadrupolar and octupolar confinements, confirms that intershell motion of the ions is less likely than intrashell motion, as already observed in molecular dynamics simulations of trapped ion clusters. ${ }^{11,21-23}$ A more detailed statistical analysis of the transition states reveals that intrashell barriers are also more symmetric and the pathways less cooperative than for intershell rearrangements. A kinetic transition network representation of the kinetics, obtained by discrete path sampling, indicates that relaxation between the main funnels is essentially Arrhenius in character, deviations appearing only at high temperatures.

The landscapes of the ion clusters were generally found to have some hierarchical character indicative of an intermediate level of frustration. This structure is mostly an effect of the long-range of the Coulomb and trap potentials, which also explains the rather low cooperativity and even the shape of individual pathways, with steep barriers but flat minima. All these features are very different from those observed in systems bound by short-range forces. ${ }^{63}$

The perfectly isotropic traps considered in the present work were chosen as archetypal models of more realistic electromagnetic confinement. In particular, the micromotion arising in radiofrequency traps was neglected here, but with a significant amplitude it could affect the relative stability of the various isomers by changing their free energy. Linear octupolar traps, which are used in practice to store large ion clouds, ${ }^{30}$ are quadrupolar along one axis, and this arrangement gives rise to tubular structures in which the thermodynamics are primarily driven by the octupolar character. $^{34}$

Shorter ranged potentials, such as the Yukawa interaction between dusty plasmas, should have interestingly distinct landscapes, and it is unclear whether the strong separation between intrashell and intershell motions would be preserved. Another possible avenue for future research could involve binary clouds, with ions carrying different or identical charge/mass ratios. Spontaneous phase separation was observed experimentally for such systems, ${ }^{16}$ and molecular dynamics simulations ${ }^{66}$ suggest a nontrivial dependence on composition. The landscapes of these ion clusters may therefore be significantly more complex than those reported here for one-component systems.
${ }^{1}$ D. H. E. Dubin and T. M. O'Neil, Rev. Mod. Phys. 71, 87 (1999).

${ }^{2}$ C. Champenois, J. Phys. B 42, 154002 (2009).

${ }^{3}$ D. J. Wineland, R. E. Drullinger, and F. L. Walls, Phys. Rev. Lett. 40, 1639 (1978).

${ }^{4}$ F. Diedrich, E. Peik, J. M. Chen, W. Quint, and H. Walther, Phys. Rev. Lett. 59, 2931 (1987).

${ }^{5}$ M. Drewsen, C. Brodersen, L. Hornekær, J. S. Hangst, and J. P. Schiffer, Phys. Rev. Lett. 81, 2878 (1998).

${ }^{6}$ W. M. Itano, J. J. Bollinger, J. N. Tan, N. Jelenkovic, X.-P. Huanh, and D. J. Wineland, Science 279, 686 (1998).

${ }^{7}$ S. Bize, S. A. Diddams, U. Tanaka, C. E. Tanner, W. H. Oskay, R. E. Drullinger, T. E. Parker, T. P. Heavner, S. R. Jefferts, L. Hollberg, W. M. Itano, and J. C. Berqquist, Phys. Rev. Lett. 90, 150802 (2003).

${ }^{8}$ M. Chwalla, J. Benhelm, K. Kim, G. Kirchmair, T. Monz, M. Riebe, P. Schindler, A. S. Villar, W. Hänsel, C. F. Roos, R. Blatt, M. Abgrall, G. Santarelli, G. D. Rovera, and P. Laurent, Phys. Rev. Lett. 102, 023002 (2009).

${ }^{9}$ O. Arp, D. Block, A. Piel, and A. Melzer, Phys. Rev. Lett. 93, 165004 (2004).

${ }^{10}$ M. Bonitz, D. Block, O. Arp, V. Golubnychiy, H. Baumgartner, P. Ludwig, A. Piel, and A. Filinov, Phys. Rev. Lett. 96, 075001 (2006).

${ }^{11}$ F. Calvo and E. Yurtsever, Eur. Phys. J. D 44, 81 (2007).

${ }^{12}$ J. P. Schiffer, Phys. Rev. Lett. 88, 205003 (2002).

${ }^{13}$ M. Drewsen and A. Brøner, Phys. Rev. A 62, 045401 (2000).

${ }^{14}$ A. Mortensen, E. Nielsen, T. Matthey, and M. Drewsen, Phys. Rev. Lett. 96, 103001 (2006).

${ }^{15}$ H. Totsuji, T. Kishimoto, C. Totsuji, and K. Tsuruta, Phys. Rev. Lett. 88, 125002 (2002).

${ }^{16}$ L. Hornakær, N. Kjaergaard, A. M. Thommesen, and M. Drewsen, Phys. Rev. Lett. 86, 1994 (2001).

${ }^{17}$ R. Rafac, J. P. Schiffer, J. S. Handst, D. H. Dubin, and D. J. Wales, Proc. Natl. Acad. Sci. U.S.A. 88, 483 (1991).

${ }^{18}$ R. W. Hasse and V. V. Avilov, Phys. Rev. A 44, 4506 (1991).

${ }^{19}$ R. A. Beekman, M. R. Roussel, and P. J. Wilson, Phys. Rev. A 59, 503 (1999).

${ }^{20}$ Z.-X. Gong, G.-D. Lin, and L.-M. Duan, Phys. Rev. Lett. 105, 265703 (2010).

${ }^{21}$ S. W. S. Apolinario and F. M. Peeters, Phys. Rev. E 76, 031107 (2007).

${ }^{22}$ J. A. Drocco, C. J. O. Reichhardt, C. Reichhardt, and B. Jankó, Phys. Rev. E 68, 060401(R) (2003).

${ }^{23}$ E. Yurtsever, F. Calvo, and D. J. Wales, Phys. Rev. E 72, 026110 (2005).

${ }^{24}$ D. J. Wales and A. M. Lee, Phys. Rev. A 47, 380 (1993).

${ }^{25}$ R. Otto, J. Mikosch, S. Trippel, M. Weidemüller, and R. Wester, Phys. Rev. Lett. 101, 063201 (2008).

${ }^{26}$ J. Walz, I. Siemers, M. Schubert, W. Neuhauser, R. Blatt, and E. Teloy, Phys. Rev. A 50, 4122 (1994).

${ }^{27}$ K. Okada, K. Yasuda, T. Takayanagi, M. Wada, H. A. Schuessler, and S. Ohtani, Phys. Rev. A 75, 033409 (2007).

${ }^{28}$ F. Calvo, C. Champenois, and E. Yurtsever, Phys. Rev. A 80, 063401 (2009).

${ }^{29}$ J. Cioslowski, J. Chem. Phys. 133, 234902 (2010); J. Cioslowski and E. Grzebielucha, ibid. 134, 124305 (2011).

${ }^{30}$ K. Okada, T. Takayanagi, M. Wada, S. Ohtani, and H. A. Schuessler, Phys. Rev. A 80, 043405 (2009).

${ }^{31}$ D. J. Wales, Energy Landscapes (Cambridge University Press, Cambridge, 2003).

${ }^{32}$ D. J. Wales and T. V. Bogdan, J. Phys. Chem. B 110, 20765 (2006).

${ }^{33}$ D. J. Wales, Curr. Opin. Struct. Biol. 20, 3 (2010).

${ }^{34}$ E. Yurtsever, E. D. Onal, and F. Calvo, Phys. Rev. A 83, 053427 (2011).

${ }^{35}$ D. J. Wales and J. P. K. Doye, J. Phys. Chem. A 101, 5111 (1997).

${ }^{36} \mathrm{Z}$. Li and H. A. Scheraga, Proc. Natl. Acad. Sci. U.S.A. 84, 6611 (1987).

${ }^{37}$ S. A. Trygubenko and D. J. Wales, J. Chem. Phys. 120, 2082 (2004).

${ }^{38}$ G. Henkelman and H. Jónsson, J. Chem. Phys. 111, 7010 (1999).

${ }^{39}$ G. Henkelman, B. P. Uberuaga, and H. Jónsson, J. Chem. Phys. 113, 9901 (2000)

${ }^{40}$ G. Henkelman and H. Jónsson, J. Chem. Phys. 113, 9978 (2000).

${ }^{41}$ J. M. Carr, S. A. Trygubenko, and D. J. Wales, J. Chem. Phys. 122, 234903 (2005).

${ }^{42}$ L. J. Munro and D. J. Wales, Phys. Rev. B 59, 3969 (1999).

${ }^{43}$ Y. Kumeda, L. J. Munro, and D. J. Wales, Chem. Phys. Lett. 341, 185 (2001).

${ }^{44}$ D. J. Wales, Int. Rev. Phys. Chem. 25, 237 (2006).

${ }^{45}$ D. J. Wales, Mol. Phys. 100, 3285 (2002).

${ }^{46}$ D. J. Wales, Mol. Phys. 102, 891 (2004). 
${ }^{47}$ D. J. Wales, OPTIM, A program for optimising geometries and calculating pathways, see http://www-wales.ch.cam.ac.uk/software.html.

${ }^{48}$ D. J. Wales, PATHSAMPLE, A program for generating connected stationary point databases and extracting global kinetics, see http://www-wales.ch.cam.ac.uk/software.html.

${ }^{49}$ F. Calvo, J. P. K. Doye, and D. J. Wales, J. Chem. Phys. 115, 9627 (2001).

${ }^{50}$ J. M. Carr and D. J. Wales, Phys. Chem. Chem. Phys. 11, 3341 (2009).

${ }^{51}$ P. A. Braier, R. S. Berry, and D. J. Wales, J. Chem. Phys. 93, 8745 (1990).

${ }^{52}$ J. P. K. Doye, D. J. Wales, and R. S. Berry, J. Chem. Phys. 103, 4234 (1995).

${ }^{53}$ D. J. Wales, Science 293, 2067 (2001).

${ }^{54}$ J. Cioslowski and E. Grzebielucha, Phys. Rev. E 78, 026416 (2008).

${ }^{55}$ O. M. Becker and M. Karplus, J. Chem. Phys. 106, 1495 (1997).

${ }^{56}$ D. A. Evans and D. J. Wales, J. Chem. Phys. 118, 3891 (2003).
${ }^{57}$ F. Calvo, F. Spiegelman, and D. J. Wales, J. Chem. Phys. 118, 8754 (2003).

${ }^{58}$ S. Krivov and M. Karplus, J. Chem. Phys. 117, 10894 (2002).

${ }^{59}$ J. P. K. Doye, M. A. Miller, and D. J. Wales, J. Chem. Phys. 111, 8417 (1999).

${ }^{60}$ D. J. Wales, M. A. Miller, and T. R. Walsh, Nature (London) 394, 758 (1998).

${ }^{61}$ S. A. Trygubenko and D. J. Wales, J. Chem. Phys. 120, 2082 (2004).

${ }^{62}$ S. A. Trygubenko and D. J. Wales, J. Chem. Phys. 121, 6689 (2004).

${ }^{63}$ F. Calvo, J. P. K. Doye, and D. J. Wales, "Energy landscapes of colloidal clusters: thermodynamics and rearrangement mechanisms," Nanoscale (in press).

${ }^{64}$ E. W. Dijkstra, Numer. Math. 1, 269 (1959).

${ }^{65}$ C. A. Angell, J. Non-Cryst. Solids 131-133, 13 (1991).

${ }^{66}$ T. Matthey, J. P. Hansen, and M. Drewsen, Phys. Rev. Lett. 91, 165001 (2003). 
The Journal of Chemical Physics is copyrighted by the American Institute of Physics (AIP). Redistribution of journal material is subject to the AIP online journal license and/or AIP copyright. For more information, see http://ojps.aip.org/jcpo/jcper/jsp 